\title{
The Self-Absorption Effect of Ni-63 Beta Source to the Silicon Carbide based Betavoltaic Battery
}

\author{
Swastya Rahastama ${ }^{a,}{ }^{,}$, Yohannes Dwi Saputra $^{a}$, Abdul Waris $^{\mathrm{b}}$ \\ anstitut Teknologi Kalimantan, Soekarno Hatta km.15 \\ bInstitut Teknologi Bandung, Ganesha No.10 \\ *Email: swastya.r@lecturer.itk.ac.id
}

(Diterima 25 Agustus 2020; Disetujui 17 Desember 2020; Dipublikasikan 29 Desember 2020)

\begin{abstract}
A typical planar structure is the most feasible conceptual design of betavoltaic battery due to its simplicity. The self-absorption of beta source, however, causes a limitation to the geometrical efficiency. Herein, we tried to investigate the self-absorption event in $\mathrm{Ni}-63$ beta source by changing the geometrical aspects and evaluated its effect on each layer of a $4 \mathrm{H}-\mathrm{SiC}$ semiconductor as the radiation-electricity converter. The design configuration from previous literature was adopted and the model was developed using Monte Carlo N-Particle X (MCNPX) consists of radioisotope source, semiconductor, and also ohmic contacts. The energy of beta emission was adjusted to the actual $\mathrm{Ni}-63$ beta spectra with an isotropic distribution of ejected particles. The average beta energy deposition degrades along with the addition of source mass thickness, but the $\mathrm{n}+$ substrate has a unique result where a peak is observed at $0.1246 \mathrm{mg} / \mathrm{cm}^{2}$ due to the selfabsorption effect. Furthermore, the rectangular surface area magnification gives a positive impact on the beta energy deposition up to $2.48 \%$ and the photon average energy deposition up to $137.21 \%$. The results of average electron absorbed dose are consistent with Oldano-Pasquarelli semi-empirical theory of selfabsorption in the beta source, where the upper layer receives a wider angular distribution of particles compared to the lower one, which corresponds to the counting geometrical coefficients.
\end{abstract}

Keywords: betavoltaic, Ni-63, radioisotope source, $\mathrm{SiC}$, self-absorption

\section{Introduction}

Nuclear microbattery has been extensively developed in the last decades to support cardiac pacemakers and other micro-electromechanical systems (MEMS). Since the first introduction of the betavoltaic battery by Paul Rappaport in 1954, however, several issues still remain and not having a significant improvement [1]. One of the major issues is how to manage a reliable safety of a high energy density source into a small package along with having an efficient device performance as ideal energy storage. At least to improve its electrical performance, a direct conversion nuclear battery can be optimized mainly in its three critical aspects: (1) radioisotope source, (2) radiation-electricity converter, (3) charge collector device.

Self-absorption or self-scattering loss is one of the main issues found in a relatively thick betaemitting source. It turns out to bring a noticeable impact on a betavoltaic device with a low beta energy radioactive material, such as Ni-63 or H-3 [2]. This behavior arises due to several factors, including the material density, the chemical compound, the structural parameter, the distribution of atoms, and the beta spectra of the material itself [3]. However, Ni-63 and $\mathrm{H}-3$ are among the most promising source for the betavoltaic device, since they both have longevity on half-life and decay only through beta emission. Related to its simplest chemical formation, the solid form of Ni-63 offers a less harmful and reliable safety of radioactive leakage to micro-battery package than $\mathrm{H}-3$ which naturally exists in gaseous form.

The experimental proofs of beta source selfabsorption have been reported from several studies [4-6]. To support those experimental results, the prediction of betavoltaic optimum design can be done through a simulation study with the aid of the Monte Carlo principle. There have been several betavoltaic simulations derived from the calculation of energy deposition through various Monte Carlo based code, such as MCNP [2,7], Geant4 [8], Penelope [9], etc. Some of these previous modeling results, however, did not consider or inform the self-absorption effect of the beta source. The optimum thickness according to the beta fluence with different shapes of the betavoltaic device had also been predicted [10], although the silicon-based betavoltaic design was 
simplified. Furthermore, there was no information about the photonic generation and energy deposited by the bremsstrahlung radiation, and often to be neglected throughout the simulations.

From the energy converter perspective, even though silicon-based semiconductor is widely available and well-studied, it is limited to its poor performance in converting the beta energy into electricity [11]. Moreover, it could not sustain a long term radiation exposure which is found since the early days of betavoltaic demonstration [12]. In the latest betavoltaic design, a wide band-gap (WBG) semiconductor such as Silicon Carbide ( $\mathrm{SiC}$ ) and Gallium Nitride ( $\mathrm{GaN}$ ) have been extensively used and considered to be the best choice for the beta to electricity converter than conventional silicon cell [13]. To further increase its electrical performance, a passivation layer or radioluminescence material can be introduced to the battery design [14-16].

Besides searching for the best material to convert radiation-electricity, better conversion efficiency of betavoltaic can be achieved by taking advantage of the geometrical aspects, including the beta source itself [17]. Even though a previous simulation from Kim et al.[10] has reported about the effect of beta source thickness on the particle fluence, there is no information about how it could affect the energy deposition which is an important parameter for betavoltaic simulation. Since the beta source is emitted isotropically in the real life, the self-absorption effect can also influence to the energy deposition if the surface area is modified.

In this study, we tried to simulate a betavoltaic device using a Ni-63 radioisotope source embedded with a $4 \mathrm{H}-\mathrm{SiC}(\mathrm{p}+, \mathrm{n}, \mathrm{n}+)$ semiconductor from [18] to investigate the effect of the beta energy deposition due to the addition of Ni-63 thickness. This could be one of the physical parameters to explain how the electrical performance measured in the experiment deviates from some of the existing theoretical modelings. Furthermore, we also evaluated the surface area magnification to the energy deposition in each layer. The Monte Carlo N-Particle code version X (MCNPX) is used as the computational tool to calculate the beta energy deposition. In addition, we also calculated the X-ray energy deposited in the respective layers and the average beta absorbed dose to give the information for further experimental consideration.

\section{Methods of Simulation}

The most basic design of a betavoltaic battery consists of a beta source, a semiconductor layer, and contacts on the front and rear surface to collect the generated carrier. Under the beta illumination, the electron-hole pairs (EHPs) will be generated inside the semiconductor, much like the photovoltaic effect. These EHPs generation causes a disturbance in thermal equilibrium and will be swept away by the built-in electric field.

To investigate the generation rate, we need to know how the beta particle interaction occurred inside the absorber. Unlike photon which undergoes only the scattering event, the beta particle trajectory is also affected by the Coulomb interaction and can be easily deflected when it enters the nuclear radius of the absorber. As a consequence, the beta particle has a weaker penetration ability compared to electromagnetic radiation. Along its path, the beta kinetic energy is mostly transferred to the atomic orbital and the nucleus of the absorber via orbital collision $\left(-\frac{d E}{d s}\right)_{c o l}$ and radiative interaction $\left(-\frac{d E}{d s}\right)_{\text {rad }}$, respectively. The total degradation of beta particle kinetic energy can be written as [19]:

$$
\left(-\frac{d E}{d s}\right)_{t o t}=\left(-\frac{d E}{d s}\right)_{c o l}+\left(-\frac{d E}{d s}\right)_{r a d}
$$

The full beta decay spectrum of Ni-63 can be seen in Figure 1. This beta decay spectrum is used to define the Ni-63 beta source in simulation based on Monte Carlo methods. From the decay scheme in [20], there is no other gamma emission nor internal conversion occurred in the process. However,

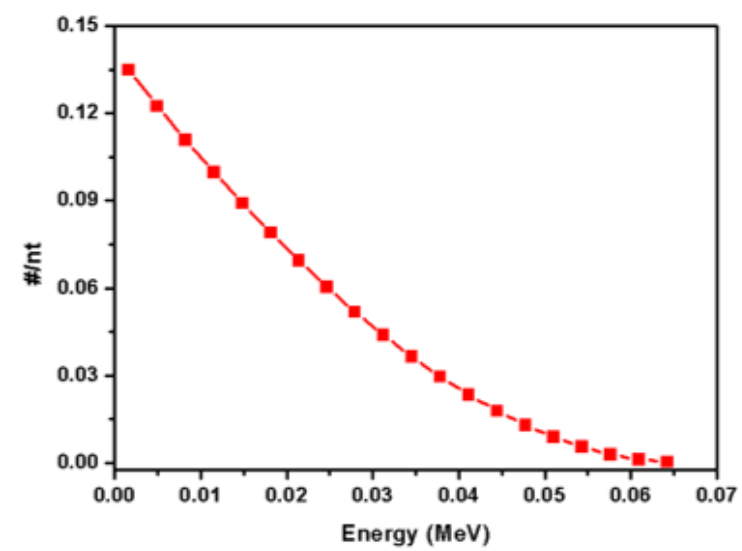

Figure 1. The beta spectra of $\mathrm{Ni}-63$ decays to $\mathrm{Cu}-63$ [22]. 
unlike alpha particles which are emitted in discrete energy, the beta particle has a continuous energy spectrum extending from zero to its maximum value. According to Fermi's theory, the beta decay is followed by a massless and chargeless particle known as neutrino or anti-neutrino [21]. For Ni-63, the beta particle emitted with an average and maximum kinetic energy of $0.0171 \mathrm{MeV}$ and 0.0667 $\mathrm{MeV}$, respectively [20].

In this study, we tried to evaluate the existing betavoltaic from previous literature, using $(\mathrm{p}+, \mathrm{n}$, $\mathrm{n}+$ ) homoepitaxy layers with $4 \mathrm{H}-\mathrm{SiC}$ n-type based substrate from Guo et al. experimental works [18]. The planar structure of the betavoltaic battery is shown in Figure 2, in which the shape of the rectangular surface will be modified along with the optimization of Ni-63 source thickness corresponds to the self-absorption effect. However, due to the lack of information about the properties of the Ni63 beta source, we assumed that it consists of a pure Ni-63 without other isotopes, which is usually done for other simulation works [23-25]. The difference might not be significant as the density of the product $(\mathrm{Cu}-63)$ is close to the decaying isotopes. Besides, the isotopic consideration only gives a slight difference to the nuclear dimensional

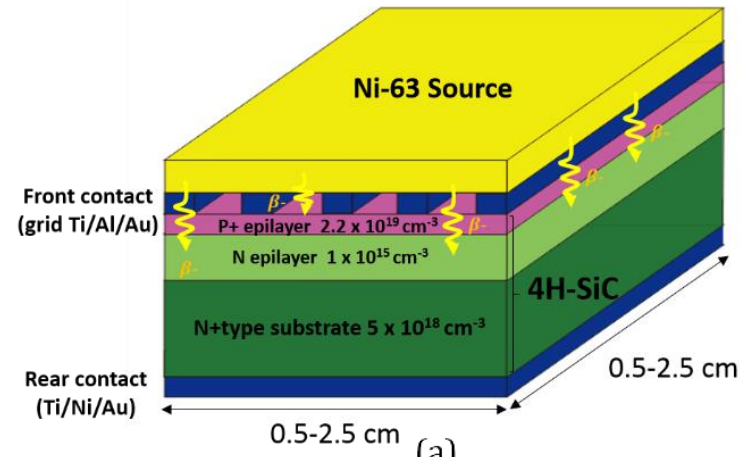

(a)

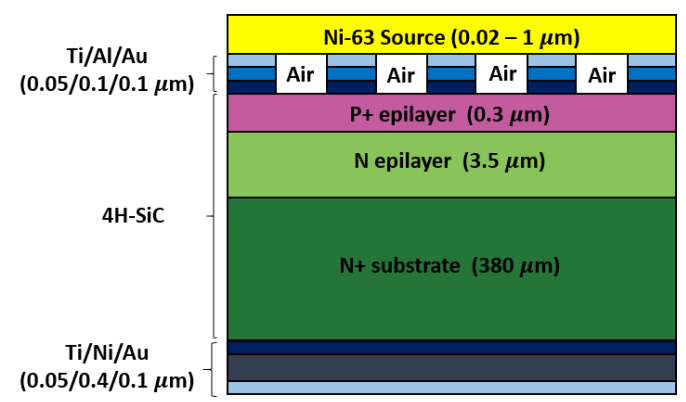

(b)

Figure 2. The geometrical structure of a basic planar betavoltaic battery consists of a pure Ni-63, $4 \mathrm{H}-\mathrm{SiC}(\mathrm{p}+, \mathrm{n}, \mathrm{n}+)$ semiconductor, and ohmic contacts used in simulation: (a) 3D view (b) crosssectional view. scale since $R \propto A^{1 / 3}$. The asumption can be made since it is not giving any effect to total cross section nor to the collisional and radiative stopping power.

MCNPX is one of the particle simulation tools based on the Monte Carlo transport principle which allows the user to simulate the interaction of a wide set of particles, including photon and electron. It also provides many calculations in a form of a tally function. Most of the simulation works in betavoltaic battery frequently used MCNP to calculate the beta energy deposition in semiconductor cell, which is subsequently used to calculate the generation rate of EHPs. The actual flux of beta particle coming to the surface of semiconductor might be different from the calculation from specific activity as the selfabsorption effect could reduce the particle flux, particularly for a low energy beta source. The absorbed dose of the beta particle is practically more useful to determine how the self-absorption affects the condition of the particle inside the semiconductor, rather than having only the particle which entered the surface. Hence, it is necessary to calculate the absorbed dose in semiconductor layers, which is formulated as [26]:

$$
D=\frac{d E}{d m}
$$

The electron steps for each random walk can be estimated with:

$$
E_{n-1}-E_{n}=-\int_{s_{i}}^{s_{i+1}}\left(\frac{d E}{d s}\right)_{t o t} d s
$$

The tally function F6 provided by MCNPX was used to determine the energy deposition for a given particle at specific cells we were interested in. The total energy deposition which is calculated with MCNP is given by:

$$
E_{D}=\frac{\rho_{a}}{\rho_{g}} \int_{V} \int_{t} \int_{E} H(E) \Phi(r, E, t) d E d t \frac{d V}{V}
$$

where $\rho_{a}$ and $\rho_{g}$ is an atom and gram density of the cell, $H(E)$ is the heating response, and $\Phi(r, E, t)$ is the particle flux which is equal to the particle velocity $v$ times the particle density $N(r, E, t)$.

The physical properties of each part of the betavoltaic cell must be given to give a valid input to the MCNPX, such as density and the atomic fraction, which are listed in Table 1 . However, the level of doping concentration at the respective $4 \mathrm{H}$ SiC homoepitaxy layers is much lower than the atomic concentration of $\mathrm{SiC}$, thus the difference in 
Table 1. The physical properties of the active cells of betavoltaic from the compendium of material data in [27].

\begin{tabular}{|c|c|c|}
\hline Materials & $\begin{array}{l}\text { Density } \\
\left(\mathrm{g} / \mathrm{cm}^{3}\right)\end{array}$ & Atomic Fraction \\
\hline $\mathrm{Ni}-63$ & 8.902 & $100 \% \mathrm{Ni}-63$ Isotope \\
\hline $\mathrm{SiC}$ & 3.21 & $\begin{array}{l}50 \% \text { Natural Si } \\
50 \% \text { Natural C }\end{array}$ \\
\hline $\mathrm{Ti}$ & 4.54 & $100 \%$ Natural Ti \\
\hline $\mathrm{Ni}$ & 8.902 & $100 \%$ Natural $\mathrm{Ni}$ \\
\hline $\mathrm{Al}$ & 2.698 & $100 \%$ Natural Al \\
\hline $\mathrm{Au}$ & 19.32 & $100 \%$ Natural $\mathrm{Au}$ \\
\hline Air (dry) & 0.0012 & $\begin{array}{l}0.015 \% \text { Natural C } \\
78.443 \% \text { Natural N } \\
21.075 \% \text { Natural O } \\
0.467 \% \text { Natural Ar }\end{array}$ \\
\hline
\end{tabular}

density and atomic fraction due to the doping concentration of $\mathrm{p}+, \mathrm{n}$, and $\mathrm{n}+$ layers of the $4 \mathrm{H}-\mathrm{SiC}$ semiconductor can be neglected. Since MCNPX is mostly based on the probability density of heating response, the calculation would not give a meaningful difference. This assumption was also done in many betavoltaic performance simulations $[7,22,23]$.

\section{Results and Discussions}

The Monte Carlo simulation was carried out using the planar structure shown in Figure 2. The statistical error can not be avoided in every MC simulation. In order to verify the reliability of MC simulation, we tried to evaluate the statistical error emerged from the beta energy deposition calculation for a different number of particle used in the simulation. The beta source was set to the maximum thickness $(1 \mu \mathrm{m})$ and the surface area of $2.5 \times 2.5 \mathrm{~cm}^{2}$ since it will produce the highest error corresponds to a wider distribution of random particle generation.

The statistical error decreased as the NPS is added exponentially. On the other hand, the higher

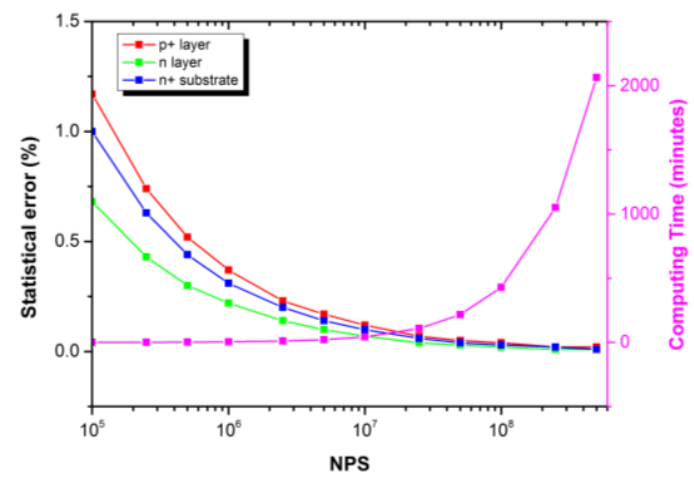

Figure 3. The statistical error and computing time of MC simulation by using different NPS.
NPS used, the longer time it needed to complete the MC simulation. As shown in Figure 3, the p+ layer produced the highest error compared to the others since it is directly exposed to the beta source which has spectra. However, at the NPS $>10^{7}$, the computation time started to grow significantly. Even though the calculation will be slightly more accurate, it is pointless to exert more than $10^{8}$ particles since the difference of error is not giving a meaningful response. More importantly, the statistical error between the three layers was already close to each other at NPS $>10^{7}$ and the values were below $0.2 \%$ which is categorized as generally reliable for MC simulation according to [19]. Therefore, we used 50 million particles in this study to have a little more accurate results $( \pm 0.04 \%$ error on average) but still with an acceptable computation time (approximately 216 minutes for each simulation).

\section{A. Energy Deposition}

As the beta particles interact with matter, some of their kinetic energy is transferred to the atoms of the absorber and scattered from its original path. For every beta particle track generated by the Monte Carlo method, the MCNP took the total energy deposition in the respective cells and averaged for each repetition of number particle history. Subsequently, the output from tally F6 is in the form of energy deposition per mass $(\mathrm{MeV} / \mathrm{g})$, which is then multiplied by the total mass of each cell to get the average energy deposition for a specific particle.

The profile of beta energy deposition in each cell are shown in Figure 4(a)-(c). It can be noticed in Fig. 4(a) and 4(b) that the addition of source mass thickness may decrease the energy deposition of each beta particle in both $\mathrm{p}+$ and $\mathrm{n}$ epilayer. It could be the first indication of the self-absorption effect happening in the simulation since the energy of the beta particle is reduced gradually due to the dissipation as it travels throughout the source.

However, there is a peak observed which is unique only to the $n+$ substrate. Hence, we obtained that the optimum mass thickness for Ni-63 is about $0.1246 \mathrm{mg} / \mathrm{cm}^{2}$ as the beta energy deposited over the surface per particle reaches its maximum value. If the source is remarkably thin, the energy of the beta particle coming to the surface is close to the spectra, since the self-absorption is minimum. However, according to the data from Kieffer et al. [28], the total electron collision cross-section at 

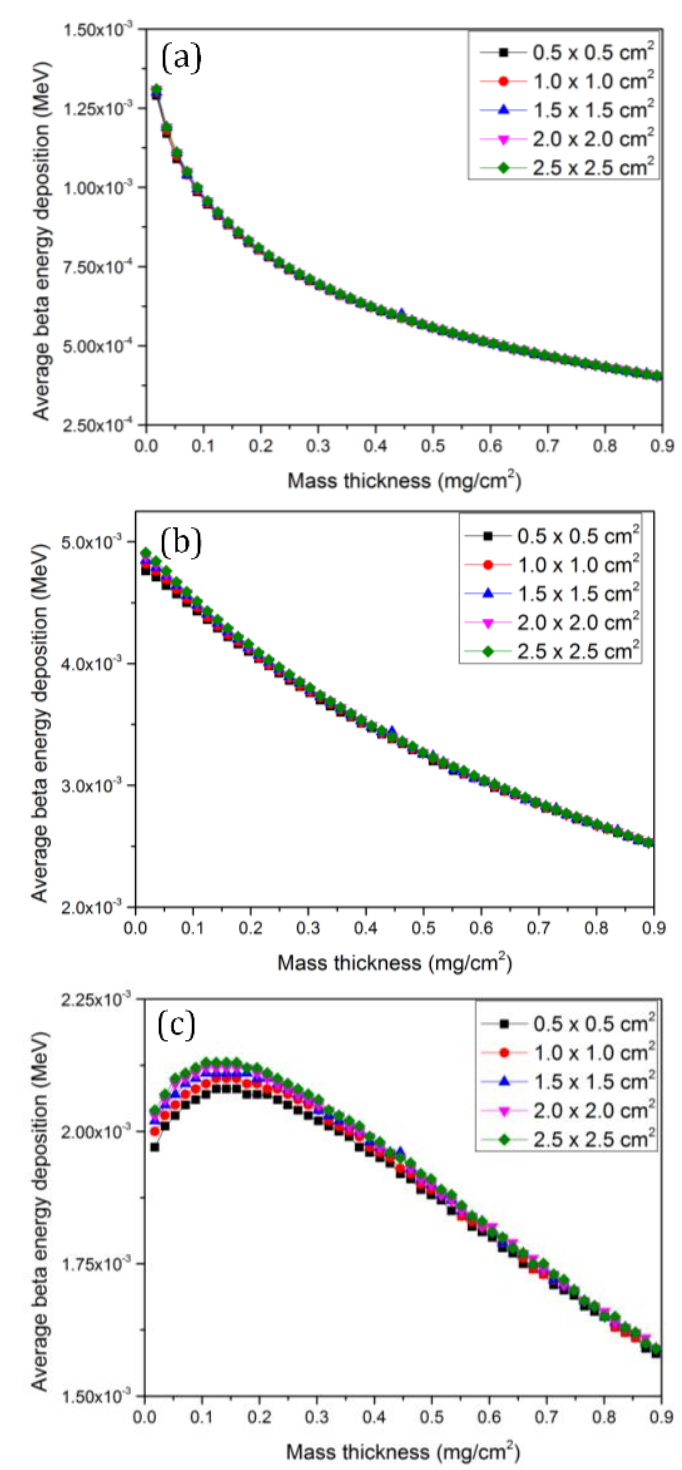

Figure 4. The average beta energy deposition inside the 4H-SiC p+-n-n+ semiconductor: (a) p+ epilayer, (b) $n$ epilayer, (c) $n+$ substrate with different surface interface area.

high energy is small as well as the low energy. The total cross-section defines how the interaction of beta particles will occur in the absorber. The data in [28] shows a similar manner to Figure 4.c, where there is a peak of the optimum cross-section for the electronic collision. This total cross-section $\sigma_{T}(E)$ is directly related to the heating response for a given particle in MCNPX, which is given by:

$$
H(E)=\sigma_{T}(E) H_{\text {avg }}(E)
$$

Otherwise, if it has a massive thickness, some of the beta particles already spent their energy on the collision in the source itself. As a consequence, the beta particle reaching the $\mathrm{n}+$ substrate is weakened on average. Beyond its optimum thickness, the average beta energy deposited will be reduced even further as the thickness is increased.

The results of average beta energy deposition at the semiconductor layers agree well with the Hui Gou et al. betavoltaic experiment, where they used $\mathrm{Ni}-63$ source with 0.096 and $0.16 \mathrm{mCi}$ activity. If we roughly calculated the mass thickness of $\mathrm{Ni}-63$ source from its specific activity, the isotope has 0.0105 and $0.0176 \mathrm{mg} / \mathrm{cm}^{2}$ for the activity of 0.096 and $0.16 \mathrm{mCi}$, respectively. As implied in several betavoltaic theoretical studies $[15,22,29]$, the model for generation rate is proportional to the incident flux ( $G \propto \Phi_{\beta}$ ). The maximum beta flux coming to the surface is the level of radioactivity. However, the experimental results from [18] show that the maximum power output can be increased up to 2.286 times (from $0.14 \mathrm{nW}$ to $0.32 \mathrm{nW}$ ), where the model implies that it could only go up to 1.667 times. Figure 4 (c) implies that the mass thickness used in Guo's experiment is less than the optimum value. The self-absorption effect may also bring a positive impact as it could lower beta energy to a value near the maximum total cross-section, thus the average beta energy deposition is increased. Hence, the betavoltaic from Guo's experiment can still be improved through the geometrical aspect of the Ni-63 source.

Furthermore, the Ni-63 rectangular surface area modification could influence energy deposition as well. This effect can be observed clearly in Figure 4(a), where the energy deposited in the $n+$ substrate gradually increases following the surface area magnification. Compared to $0.5 \mathrm{x}$ $0.5 \mathrm{~cm}^{2}$, the energy deposited in $\mathrm{n}+$ substrate is enhanced up to $2.48 \%$ for $2.5 \times 2.5 \mathrm{~cm}^{2}$ surface area. On the other hand, the p-type and n-type layers may also gain a slight improvement but not as much as energy deposition in the $n+$ substrate. Since the source is emitted isotropically, the variation of the surface area gives a different value of space integration parameter which modifies the attainment of beta flux over the interface. Even though the active cell only receives a minor incremental, thus if we want to design a microscaled area, the deterioration of electrical performance can be neglected.

This beta energy deposition could influence the electrical performance of betavoltaic since it is the primary mechanism for the ionization event to produce EHPs. Furthermore, a single beta particle from Ni-63 ejected with an average of $17.1 \mathrm{KeV}$ is 
responsible to generate multiple EHPs inside the semiconductor. The minimum energy to produce an EHP can be described as follows [6]:

$$
E_{\text {min }}=\frac{2 m_{e}+m_{h}}{m_{e}+m_{h}} E_{g}
$$

However, the kinetic energy of the beta particle is not directly converted to generate carriers. The total energy dissipated for each interaction of beta particle to produce EHP is given by[23]:

$$
\varepsilon=\frac{14}{5} E_{g}+\hbar \omega_{R}=2.8 E_{g}+0.5
$$

where $E_{g}$ is the band-gap energy of converter (about 3.2-3.3 eV for $\mathrm{SiC}$ ), and $\hbar \omega_{R}$ is the Raman quanta. For a typical $4 \mathrm{H}-\mathrm{SiC}$ based material, the
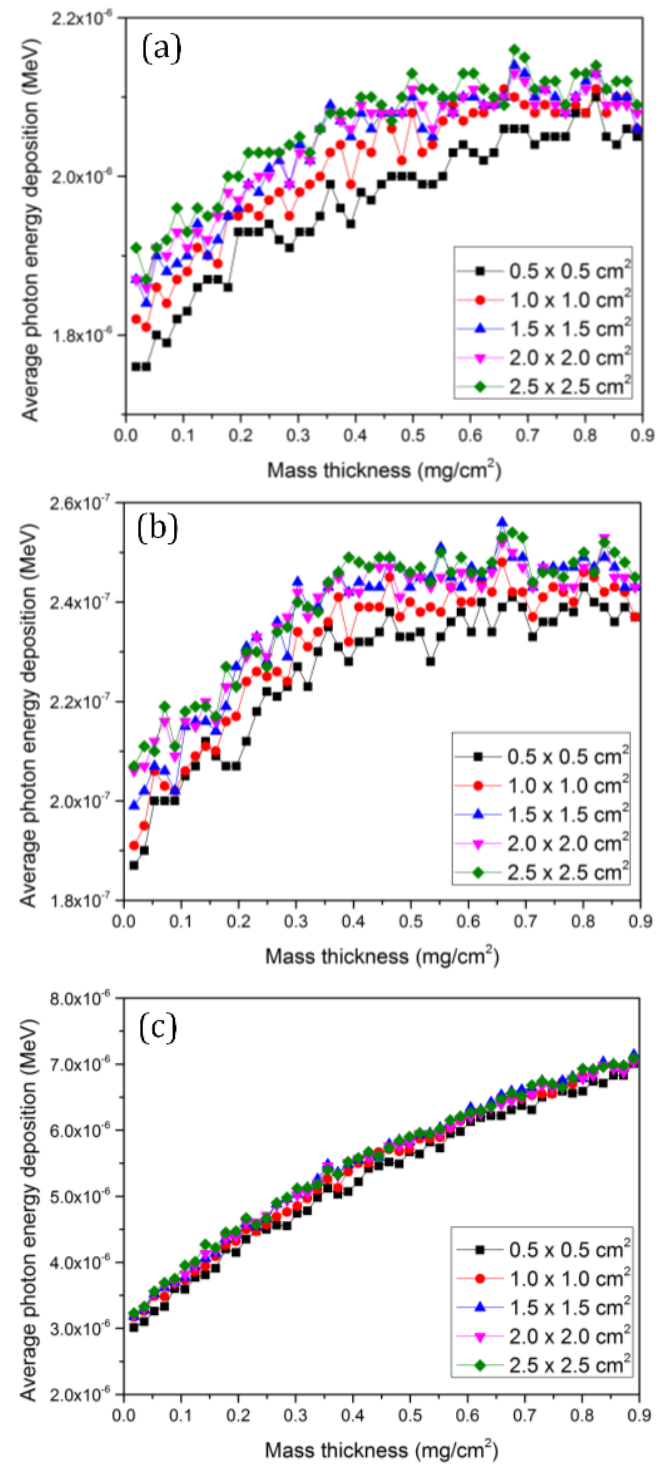

Figure 5. The average photon energy deposition inside the 4H-SiC p+-n-n+ semiconductor: (a) p+ epilayer, (b) $\mathrm{n}$ epilayer, (c) $\mathrm{n}+$ subtrate with different surface interface area. total energy dissipated for each EHP generation about 9.74-10.24 eV.

The photonic emission due to the internal bremsstrahlung effect may also take a role as a tertiary, indirect mechanism to produce additional EHPs. The bremsstrahlung radiation could also be produced by the secondary electron [30]. Figure 5 (a)-(c) show the average photon energy deposition per particle for a different surface area. The fluctuation of photon energy deposition emerges in the $p+$ epilayer where the cell is directly exposed to the beta source. The secondary or subsequent electrons which have a probability to induce additional bremsstrahlung radiation took part in this photonic generation. Since the beta particle encountered the first two layers left with sufficient energy to do ionization, thus much of the secondary and subsequent electron would be produced in this region. Even though the trends give rise to the photonic energy deposition as the source mass thickness is increased, it will become saturated at a certain value of source thickness.

The degradation of beta particles caused by electron-electron collision competes with the bremsstrahlung event. The ratio of radiative to collisional stopping power is given by [26]:

$$
\frac{S_{\text {rad }}}{S_{\text {col }}} \cong \frac{Z E}{800}
$$

The bremsstrahlung event depends on the total nuclear charge of the absorber and the incident beta energy. As the source thickness increased, most of the bremsstrahlung radiation produced in the source itself since $Z_{N i}>Z_{S i C}$. The first two layers become saturated quickly since they were the main production of bremsstrahlung radiation, but then it switched to the source as the thickness is increased.

According to Katz-Penfold semi-empirical equation, some of the beta particles have already been absorbed in the $\mathrm{p}+$ or $\mathrm{n}$ epilayer. The formulation of Katz-Penfold can be written as[31]:

$$
R_{\max }=\frac{0.412}{\rho} E_{\beta}^{1.265-0.0954 \ln E_{\beta}}
$$

where $\rho$ is the density of the absorber (SiC), and $E_{\beta}$ is the kinetic energy of the beta particle in MeV. As a consequence, the $n+$ substrate only gives a minor contribution to photonic emission related to a weak beta particle penetration ability. Still, the continuum spectra of beta decay allow some particles to be emitted with sufficient energy to penetrate into the $n+$ substrate. 


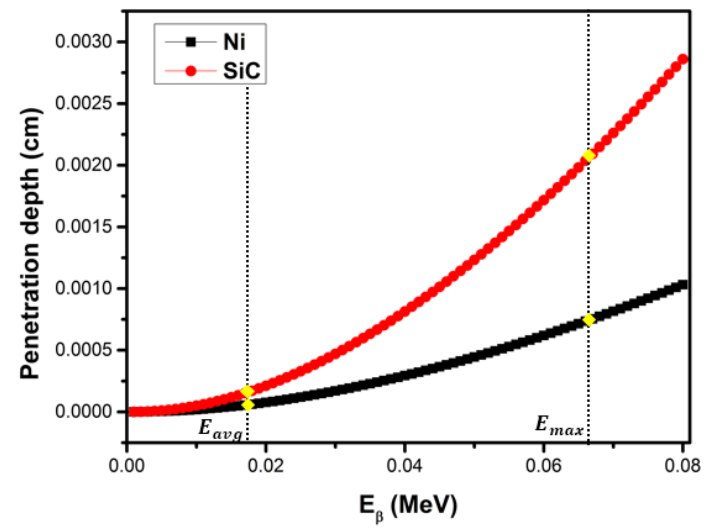

Figure 7. Maximum penetration range of beta particle in $\mathrm{Ni}$ and $\mathrm{SiC}$ using Katz-Penfold semiempirical approximation.

The plot of the Katz-penfold formula for SiC and Ni-63 absorber can be seen in Figure 6. At its $E_{\text {avg, }}$, the beta particle emitted from Ni-63 could penetrate up to $0.55 \mu \mathrm{m}$ and $1.54 \mu \mathrm{m}$ range inside nickel and $\mathrm{SiC}$, respectively. On the other hand, it could penetrate further at its $E_{\max }$ up to $7.49 \mu \mathrm{m}$ in nickel and $20.76 \mu \mathrm{m}$ in SiC. The geometrical structure of the active cell should not be thicker than the predicted maximum range of beta particles inside both materials. However, the $\mathrm{n}+$ substrate is located in a deeper region where the beta particle is only left with low energy, thus it mostly absorbing photons from other regions rather than producing them. As a result, Figure 5(c) shows a better fluctuation and not becoming saturated as quickly as other regions. The saturation may depend on the size and the optical properties of the whole betavoltaic configuration.

When the beta particle enters the nucleus of $\mathrm{Ni}$ 63 , it resulted in a larger amount of photonic emission. Consequently, a larger geometry of radioactive source means the more bremsstrahlung radiation it will produce. As shown in Figure 5 (a)(c), the area magnification improved the photonic energy deposition in $\mathrm{p}+$ epilayer, $\mathrm{n}$ epilayer, and $\mathrm{n}+$ substrate about $137.21 \%, 36.89 \%$, and $22.7 \%$, respectively. The empirical approximation of the energy yield fraction due to the electron-nuclei interaction given as [32]:

$$
Y_{i}=\frac{6 \times 10^{-4} E Z}{1+6 \times 10^{-4} E Z}
$$

As the source becomes the absorber itself, we can get a much more photon yield when we increase the source thickness.
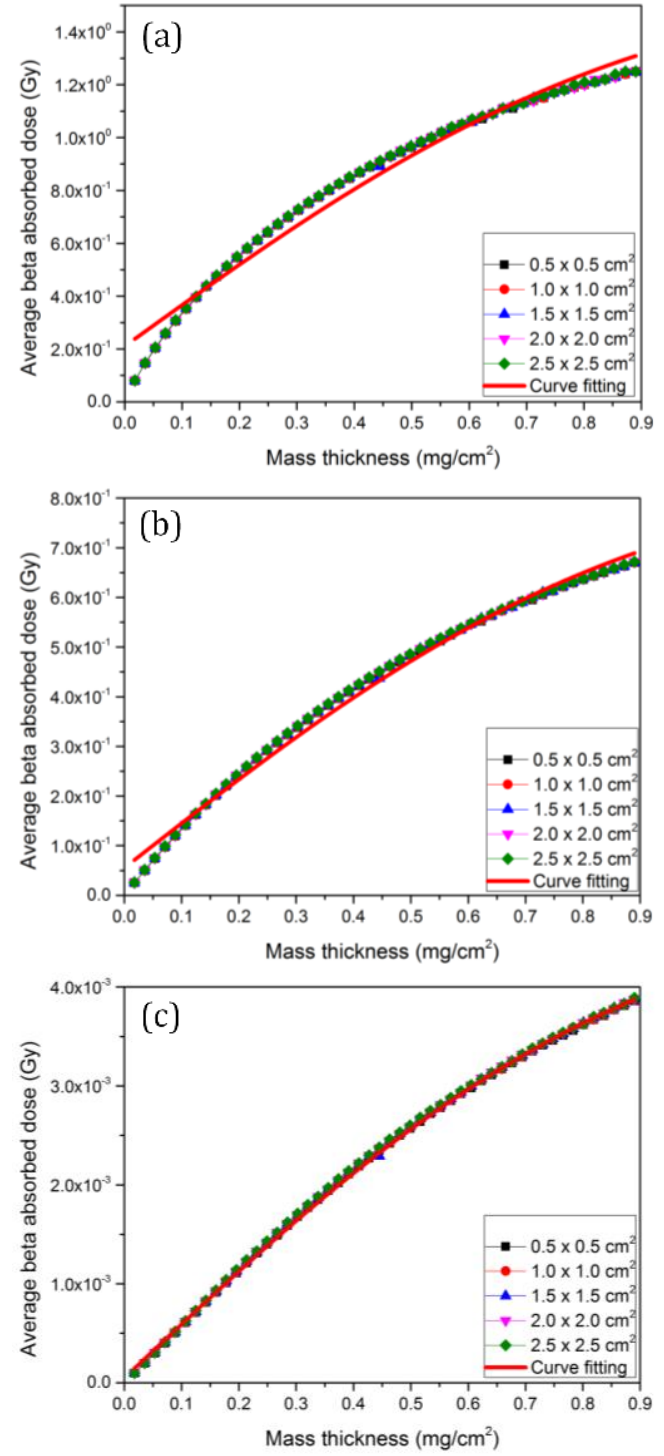

Figure 6. The average beta particle absorbed dose inside the $4 \mathrm{H}-\mathrm{SiC} \mathrm{p}+-\mathrm{n}-\mathrm{n}+$ semiconductor: (a) $\mathrm{p}+$ epilayer, (b) $n$ epilayer, (c) $n+$ substrate with different surface interface area

\section{B. Absorbed Dose}

To maintain its electrical performance, one critical factor in assuring the radiation durability of betavoltaic major components, including the conversion cells and the charge collector. Despite the fact that the beta particle energy from Ni-63 is far below the threshold of radiation damage for $\mathrm{SiC}$, however, some displacements may occur to the $\mathrm{Si}$ and $C$ sublattices [33]. It is important to calculate the absorbed dose of radiation, particularly in the converter cell, to avoid such a rapid degradation caused by intense radiation exposure.

The profile of incident flux was assumed to be 
uniformly distributed, with the total amount of emitting radiation equal to the activity of the source. Each simulation was set to timeindependent, thus the absorbed dose is instantaneous when the cell is exposed for the first time related to source radioactivity. The total radioactivity of $\mathrm{Ni}-63$ also depends on its thickness, with a specific activity of $2.097 \mathrm{GBq} / \mathrm{g}$ [34]. Hence, the total activity is varied from 0.25 to $12.55 \mathrm{mCi}$.

The average absorbed dose of the beta particle in each layer is shown in Fig 7 (a)-(c). Generally, the shape of these curves is proportional to the particle fluence dependence to Ni-63 thickness which was previously simulated by Kim et al [10]. From their report, the curve would become saturated due to the effect of self-absorption. The particle fluence does correlate with the absorbed dose, which determines the energetical condition of the beta particle as it enters the surface. Therefore, the selfabsorption effect causes a limitation to the average absorbed dose as well. The variation to the surface area does not give any severe impact on the average absorbed dose of the electron. At the optimum mass thickness, the electron average absorbed dose is about $0.397 \mathrm{~Gy}, 0.163 \mathrm{~Gy}$, and $0.00072 \mathrm{~Gy}$ in the p+ epilayer, $n$ epilayer, and $n+$ substrate, respectively.

The shape of these curves are also valid with the theoretical explanation of self-absorption and self scattering effect of beta sources from OldanoPasquarelli, which they introduced a semiempirical approximation of counting geometric efficiency given by the following equation [5]:

$$
f_{G}=G_{0}+\left(G_{1}-G_{0}\right) \phi
$$

where the constant $G_{0}$ and $G_{1}$ came from the series expansion term of $f_{G}$, are the counting geometry coefficients as a function of angular distribution $\Omega$. The slope can be described by the error integral function of $\phi$ varies from 0 to 1 , which is given by:

$$
\phi=2 \int_{0}^{\mu^{*} s} e^{-x^{2}} d x
$$

where $s$ is the thickness of source and $\mu^{*}$ is the selfabsorption coefficient corrected by OldanoPasquarelli, which can be calculated with:

$$
\mu^{*}=0.0488 E_{\max }^{-1.41}
$$

For Ni-63 with $E_{\max }=0.067 \mathrm{MeV}$, we obtained $\mu^{*}=2.206 \mathrm{~cm}^{2} / \mathrm{mg}$. The counting geometric
Table 2. The counting geometric coefficients from the self-absorption semi-empirical approximation in different layers of the $4 \mathrm{H}-\mathrm{SiC}$ semiconductor.

\begin{tabular}{|l|c|c|}
\hline $\begin{array}{c}\text { 4H-SiC cell } \\
\text { layers }\end{array}$ & $\boldsymbol{G}_{\mathbf{0}}$ & $\boldsymbol{G}_{\mathbf{1}}$ \\
\hline $\mathrm{p}+$ epilayer & 0.21024 & 1.59751 \\
\hline n epilayer & 0.055 & 0.85571 \\
\hline n+ substrate & $4.75407 \mathrm{E}-5$ & 0.00488 \\
\hline
\end{tabular}

coefficients from the electron average absorbed dose graph can also be fitted using Eq. (11), which the results are listed in Table 2. From the evaluation of both $G_{0}$ and $G_{1}$, the evidence the beta particles traveling in the upper layer have a wider angular distribution. It is obvious that only a few particles could reach the deepest layer where they mostly went straight to minimize the energy loss per depth, resulting in a lesser $G$ value and a narrow angular distribution.

\section{Conclusions}

In this present work, we have investigated the self-absorption effect of Ni-63 beta source to the $4 \mathrm{H}-\mathrm{SiC}(\mathrm{p}+, \mathrm{n}, \mathrm{n}+)$ semiconductor cells with rectangular planar design from previous literature using MCNPX. The evidence of this effect causes the measured values from the experiment to deviates from the theoretical model. From this simulation, it was found that the use of the Ni-63 source in the experiment can still be improved since it is not at the optimum peak of the mass thickness (about $0.1246 \mathrm{mg} / \mathrm{cm}^{2}$ ). The photonic energy deposition saturates quickly in the first two layers, due to the competition with collisional stopping power where the ratio is proportional to the beta energy. However, this photonic emission might be useful to generate more EHPs with an internal mechanism. As we evaluated the average beta absorbed dose, the shape of curves in each layer indicated a limiting efficiency which is consistent with the previous simulation of particle fluence from Kim et al. and the self-absorption effect semiempirical approximation. According to the fitting results from the semi-empirical formula, the distribution of beta particle in $n+$ substrate must have a narrow angular distribution, to minimize the energy loss due to atomic interaction along its way. Hence, to maximize the electrical performance, the total thickness of the converter cell should consider the maximum penetration range of beta particles. 


\section{Acknowledgments}

This research was funded by the Ministry of Research and Technology of the Republic of Indonesia, 2020.

\section{References}

[1] Krasnov, A. A. dan Legotin, S. A., Advances in the Development of Betavoltaic Power Sources (A Review), Instruments and Experimental Techniques, 63(4), pp.437-452, 2020.

[2] Alam, T. R., Pierson, M. A., dan Prelas, M. A., Beta particle transport and its impact on betavoltaic battery modeling, Applied Radiation and Isotopes, 130(August), pp.8089, 2017.

[3] Wacharasindhu, T., Composite Semiconductor-Based Micro Power Source(July), 2012.

[4] Russo, J., Litz, M. S., William Ray, I. I., Berk, H., Cho, H., Bigio, D. I., Weltz, A., dan Alam, T. R., Planar and textured surface optimization for a tritium-based betavoltaic nuclear battery, International Journal of Energy Research, 43(9), pp.4370-4389, 2019.

[5] Oldano, C. dan Pasquarelli, A., Self-absorption and self-scattering effects in the beta particles sources, Nuclear Instruments and Methods, 36(C), pp.192-196, 1965.

[6] Spencer, M. G. dan Alam, T., High power direct energy conversion by nuclear batteries, Applied Physics Reviews, 6(3), 2019.

[7] Rahastama, S. dan Waris, A., Analytical Study of $90 \mathrm{Sr}$ Betavoltaic Nuclear Battery Performance Based on p-n Junction Silicon, Journal of Physics: Conference Series, 739(1), 2016.

[8] Theirrattanakul, S. dan Prelas, M., A methodology for efficiency optimization of betavoltaic cell design using an isotropic planar source having an energy-dependent beta particle distribution, Applied Radiation and Isotopes, 127(May), pp.41-46, 2017.

[9] Alam, T. R., Spencer, M. G., Prelas, M. A., dan Pierson, M. A., Design and optimization of radioisotope sources for betavoltaic batteries, International Journal of Energy Research, 42(7), pp.2564-2573, 2018.

[10] Kim, T., Lee, N., Jung, H. K., dan Kim, J. H., Enhancement of energy performance in betavoltaic cells by optimizing self-absorption of beta particles, INTERNATIONAL JOURNAL OF ENERGY RESEARCH, 40(December 2015), pp.522-528, 2016.

[11] Alam, T. R. dan Pierson, M. A., Principles of Betavoltaic Battery Design, Journal of Energy and Power Sources, 3(1), pp.11-41, 2016.
[12] Lei, Y., Yang, Y., Li, G., Liu, Y., Xu, J., Xiong, X., Luo, S., dan Peng, T., Demonstration and aging test of a radiation-resistant strontium-90 betavoltaic mechanism, Applied Physics Letters, 116(15), 2020.

[13] Chen, C., Wang, N., San, H., dan Cheng, Z., Highefficient betavoltaic batteries using graphenecoated TiO2 nanotube arrays, Proceedings of IEEE Sensors, pp.5-7, 2017.

[14] Xu, Z., Liu, Y., Zhang, Z., Chen, W., Yuan, Z., Liu, K., dan Tang, X., Enhanced radioluminescent nuclear battery by optimizing structural design of the phosphor layer, International Journal of Energy Research, 42(4), pp.17291737, 2018.

[15] Rahastama, S., Waris, A., Viridi, S., dan Iskandar, F., Optimization of surface passivation parameters in [147Pm]-Si planar p-n junction betavoltaic based on analytical 1D minority carrier diffusion equation approaches, Applied Radiation and Isotopes, 151(September 2017), pp.226-234, 2019.

[16] Zhang, Z. R., Liu, Y. P., Tang, X. Bin, Xu, Z. H., Yuan, Z. C., Liu, K., dan Chen, W., GaAs lowenergy X-ray radioluminescence nuclear battery, Nuclear Instruments and Methods in Physics Research, Section B: Beam Interactions with Materials and Atoms, 415(September 2017), pp.9-16, 2018.

[17] Tompkins, R. P., Hogan, K., Pullen, C., Kierzewski, I., Kelley, S., Doumbia, M., Smith, B. A., Shahedipour-Sandvik, S., dan Litz, M., Comparison of Planar vs. Textured Silicon Carbide ( SiC ) Betavoltaic Devices, 2020.

[18] Guo, H., Shi, Y., Zhang, Y., Zhang, Y., dan Han, J., Fabrication of SiC p-i-n betavoltaic cell with $63 \mathrm{Ni}$ irradiation source, 2011 IEEE International Conference of Electron Devices and Solid-State Circuits, EDSSC 2011, 2011.

[19] M. Navabpour, B. M., A general Monte Carlo NParticle transport code, LA12625-M, Version B, 4(December), pp.2-27, 2004.

[20] Lee, K. B., Ni-63 Decay Data, Table De Radionucleides, pp.1-3, 2006.

[21] Guglinski, W., Re-evaluation of Fermi's theory of beta-decay, International Journal of Fundamental Physical Sciences, 8(2), pp.1943, 2018.

[22] Zuo, G., Zhou, J., dan Ke, G., A Simple theoretical model for $63 \mathrm{Ni}$ betavoltaic battery, Applied Radiation and Isotopes, 82pp.119-125, 2013.

[23] Gorbatsevich, A. A., Danilin, A. B., Korneev, V. I., Magomedbekov, E. P., dan Molin, A. A., Analysis (Simulation) of Ni-63 beta-voltaic cells based on silicon solar cells, Technical Physics, 61(7), pp.1053-1059, 2016. 
[24] Svintsov, A. A., Krasnov, A. A., Polikarpov, M. A., Polyakov, A. Y., dan Yakimov, E. B., Betavoltaic battery performance: Comparison of modeling and experiment, Applied Radiation and Isotopes, 137(April), pp.184-189, 2018.

[25] Liu, Y. M., Lu, J. Bin, Li, X. Y., Xu, X., He, R., dan Wang, H. D., A $4 \mathrm{H}-\mathrm{SiC}$ betavoltaic battery based on a $63 \mathrm{Ni}$ source, Nuclear Science and Techniques, 29(11), 2018.

[26] Nikjoo, Hooshang; Uehara, Suzho; Emfietzoglou, D., Interaction of Radiation with Matter, CRC Press, 2012.

[27] Customs, U. S., Protection, B., Nuclear, D., dan Office, D., Compendium of Material Composition Data for Radiation Transport Modeling, 2011.

[28] Kieffer, L. J., Low-Energy electron-Collision cross-Section data. Part II: ElectronicExcitation cross-sections, Atomic Data and Nuclear Data Tables, 1(C), pp.121-287, 1969.

[29] Liu, Y. M., Lu, J. Bin, Li, X. Y., Xu, X., He, R., Zheng, R. Z., dan Wei, G. D., Theoretical Prediction of Diamond Betavoltaic Batteries Performance Using 63Ni, Chinese Physics Letters, 35(7), 2018.
[30] Ishii, K. dan Morita, S., Internal Bremsstrahlung Production by Secondary Electrons Ejected by Proton Impact, Japanese Journal of Applied Physics, 17pp.374-376, 1978.

[31] Cheu, D. S., Adams, T. E., dan Revankar, S. T., Derivation of critical parameters of betavoltaics, International Conference on Nuclear Engineering, Proceedings, ICONE, 9pp.1-9, 2018.

[32] Amato, E., Physics for Radiation Protection, 2014.

[33] Azarov, A. Y., Titov, A. I., Karaseov, P. A., dan Hallén, A., Effect of collision cascade density on radiation damage in $\mathrm{SiC}$, Nuclear Instruments, and Methods in Physics Research, Section B: Beam Interactions with Materials and Atoms, 267(8-9), pp.1247-1250, 2009.

[34] Hunt, S., Nickel-63, Radioactive Material Safety Data Sheet, pp.1-3, 1998. 